\title{
Accuracy and generalizability of using automated methods for identifying adverse events from electronic health record data: a validation study protocol
}

Christian M. Rochefort ${ }^{1,2,3^{*}}$, David L. Buckeridge ${ }^{3}$, Andréanne Tanguay ${ }^{1}$, Alain Biron ${ }^{4,5}$, Frédérick D'Aragon ${ }^{6}$, Shengrui Wang ${ }^{7}$, Benoit Gallix ${ }^{8}$, Louis Valiquette ${ }^{9}$, Li-Anne Audet ${ }^{1}$, Todd C. Lee ${ }^{10}$, Dev Jayaraman ${ }^{10}$, Bruno Petrucci ${ }^{11}$ and Patricia Lefebvre ${ }^{4}$

\begin{abstract}
Background: Adverse events (AEs) in acute care hospitals are frequent and associated with significant morbidity, mortality, and costs. Measuring AEs is necessary for quality improvement and benchmarking purposes, but current detection methods lack in accuracy, efficiency, and generalizability. The growing availability of electronic health records (EHR) and the development of natural language processing techniques for encoding narrative data offer an opportunity to develop potentially better methods. The purpose of this study is to determine the accuracy and generalizability of using automated methods for detecting three high-incidence and high-impact AEs from EHR data: a) hospital-acquired pneumonia, b) ventilator-associated event and, c) central line-associated bloodstream infection.

Methods: This validation study will be conducted among medical, surgical and ICU patients admitted between 2013 and 2016 to the Centre hospitalier universitaire de Sherbrooke (CHUS) and the McGill University Health Centre (MUHC), which has both French and English sites. A random 60\% sample of CHUS patients will be used for model development purposes (cohort 1, development set). Using a random sample of these patients, a reference standard assessment of their medical chart will be performed. Multivariate logistic regression and the area under the curve (AUC) will be employed to iteratively develop and optimize three automated AE detection models (i.e., one per AE of interest) using EHR data from the CHUS. These models will then be validated on a random sample of the remaining $40 \%$ of CHUS patients (cohort 1, internal validation set) using chart review to assess accuracy. The most accurate models developed and validated at the CHUS will then be applied to EHR data from a random sample of patients admitted to the MUHC French site (cohort 2) and English site (cohort 3) - a critical requirement given the use of narrative data -, and accuracy will be assessed using chart review. Generalizability will be determined by comparing AUCs from cohorts 2 and 3 to those from cohort 1.

(Continued on next page)
\end{abstract}

\footnotetext{
* Correspondence: Christian.Rochefort@usherbrooke.ca

${ }^{1}$ School of Nursing, Faculty of Medicine and Health Sciences, University of

Sherbrooke, 3001, 12e Avenue Nord, Sherbrooke, QC J1H 5N4, Canada

${ }^{2}$ Centre de recherche de l'Hôpital Charles-LeMoyne, University of

Sherbrooke-Campus Longueuil, 150 Place Charles-LeMoyne, Longueuil, QC

J4K 0A8, Canada

Full list of author information is available at the end of the article
} 
(Continued from previous page)

Discussion: This study will likely produce more accurate and efficient measures of AEs. These measures could be used to assess the incidence rates of AEs, evaluate the success of preventive interventions, or benchmark performance across hospitals.

Keywords: Adverse events, Electronic health record, Acute care hospital, Automated detection, Natural language processing, Patient safety, Data warehouse

\section{Background}

Adverse events (AEs) are injuries caused by medical management rather than by the underlying condition of the patient [1]. AEs in acute care hospitals are frequent and associated with significant morbidity, mortality and costs $[2,3]$. For this reason, preventing AEs is a high priority worldwide $[4,5]$. To evaluate the success of preventive measures, there is a need for accurate, timely and efficient methods for monitoring $\mathrm{AE}$ rates [6, 7]. Moreover, with the growing emphasis on benchmarking and public reporting of $\mathrm{AE}$ data, these methods must allow for valid inter-institutional comparisons [8, 9]. However, at present, there are no such methods.

Indeed, hospitals typically rely on manual chart review, prevalence surveys, incident reporting systems or discharge diagnostic codes for monitoring AE rates [10]. Manual chart review is a time-consuming, resourceintensive and costly process $[6,11]$. As a consequence, it is an impractical means for the routine detection and hospital-wide monitoring of AEs. Prevalence surveys similarly lack in efficiency and scalability and are subject to important inter-observer variations in the reported $\mathrm{AE}$ rates $[11,12]$. Incident reporting systems are known to significantly underestimate the true incidence rate of AEs [13]. Discharge diagnostic codes have low sensitivity and positive predictive value (PPV) for detecting AEs [14]. Moreover, important variations in coding practices across institutions preclude their use for benchmarking purposes $[14,15]$. Thus, the limitations in existing methods for measuring AEs have curtailed the ability to conduct continuous quality monitoring in acute care hospitals and the capacity to benchmark performances across institutions.

With the advent of electronic health records (EHR), and the development of automated methods for encoding and classifying EHR data, an exciting opportunity has emerged to develop potentially better methods of AE detection. Moreover, with the growing adoption of standards for storing and exchanging EHR data across applications and institutions [16], there is an opportunity to develop methods of $\mathrm{AE}$ detection that are potentially generalizable; a key requirement to valid benchmarking.

Taking advantage of these new opportunities, researchers have started to develop novel and potentially more accurate and efficient methods of AE detection, such as the natural language processing (NLP) of clinical narratives $[12,17,18]$. For instance, in 2012, we received funding from the Canadian Institutes of Health Research (CIHR) to examine the accuracy of NLP techniques for identifying venous thromboembolism (VTE) from electronic narrative radiology reports. We found that NLP techniques are highly efficient and accurate in identifying this $\operatorname{AE}[19,20]$. While VTEs can be objectively detected from a single source of EHR data (i.e., narrative radiology reports), this is not the case for most AEs (e.g., pneumonia). For these events, several sources of EHR data must be combined to satisfy existing case definitions (e.g. microbiology, laboratory, radiology, vital signs) [21-23]. However, the accuracy and generalizability of such AE detection models are unknown $[6,18]$.

To move the field forward, there is a strong need to determine the accuracy of AE detection models that integrate the information from all available EHR data sources [6]. Moreover, to obtain valid interinstitutional comparisons, the generalizability of these models to other acute care hospitals, including both French and English settings-which is essential given their reliance on narrative data,- - must be established. The proposed study aims to address these requirements.

Specifically, this study aims to determine the accuracy and generalizability of using automated methods for detecting AEs from EHR data. Three AEs were selected for the purpose of this study: a) hospital-acquired pneumonia, b) ventilator-associated events and, c) central lineassociated bloodstream infection. The rationale for selecting these AEs is provided in the Methods section.

\section{Methods}

\section{Settings}

This study will be conducted at two leading Canadian academic health centres: 1) Centre hospitalier universitaire de Sherbrooke (CHUS) and, 2) McGill University Health Centre (MUHC). The CHUS is composed of two acute care hospitals and has close to 700 beds. It serves a population of 500,000 people with annual volumes of 32,000 hospitalizations, 27,000 surgical procedures and 4500 intensive care unit (ICU) admissions [24]. The MUHC is composed of three acute care hospitals, including a French site and two English sites, and has more than 800 adult beds. It serves a population of 1.7 
million people, with annual volumes of 40,000 hospitalizations, 33,300 surgical procedures and 6000 ICU admissions [25].

\section{Design and population}

The study population consists of all adult medical, surgical and ICU patients admitted to the CHUS and the MUHC between January 1, 2013 and December 31, 2016. Our proposed approach to AE detection model development and validation builds on and expand our prior research work in the area [26]. First, we will use a random $60 \%$ sample of all patients admitted to the CHUS between the aforementioned dates for model development purposes (cohort 1, development set) (Fig. 1). Then, using a random sample of these patients, a reference standard assessment of their medical chart will be performed to determine their true $\mathrm{AE}$ status (i.e., positive or negative). Using the manually reviewed cases as the reference standard, three automated $\mathrm{AE}$ detection models will be iteratively developed and optimized (i.e., one for each $\mathrm{AE}$ of interest, which are hospital-acquired pneumonia [HAP], ventilator-associated events [VAE], and central line-associated bloodstream infection [CLABSI]). These models will be developed to mirror published AE definitions (e.g., Centers for Disease Control and Prevention/National Healthcare Safety Network [CDC/NHSN] surveillance definitions) [21-23], which will also guide electronic health record (EHR) data extraction at the CHUS (Table 1). The most accurate models will then be validated on a random sample of the remaining $40 \%$ of CHUS patients (cohort 1, internal validation set), and a reference standard assessment of the medical chart will be performed (Fig. 1) [26].

To determine the extent to which these models can be generalized to other acute care settings (including both French and English hospitals) - a critical requirement given the reliance of these models on narrative data-, the most accurate models developed and validated at the CHUS will be applied to a random sample of patients admitted to the MUHC French site (cohort 2, French external validation set) and to the MUHC English sites (cohort 3, English external validation set), and a reference standard assessment of the medical chart will be performed. Prior to applying the models to data from the MUHC English sites, French narrative data employed as predictor of $\mathrm{AE}$ occurrence in the CHUS models will be translated into English using a previously validated natural language processing (NLP) approach [27].

\section{Data sources}

Data required for developing the $\mathrm{AE}$ detection models will be extracted from the CHUS and the MUHC information systems and clinical data warehouses, and will be linked by unit, patient, and hospital admission date. Specifically, data will be extracted from eight electronic databases: 1) radiology, 2) laboratory, 3) microbiology, 4) pharmacy, 5) vital signs, 6) admission, discharge, and transfer, 7) intensive care unit, and 8) hospital discharge abstracts (Table 1). Narrative data from these sources (e.g., radiology reports) will be converted to numeric using NLP techniques developed and validated in our prior research work $[19,20]$.

\section{Measures}

\section{Adverse events}

Three potentially preventable AEs were selected for the purpose of this study: a) hospital-acquired pneumonia (HAP); defined as an infection of the lung parenchyma occurring $48 \mathrm{~h}$ or more after hospital admission [21], b) ventilator-associated event (VAE); an $\mathrm{AE}$ indicator that was introduced by the CDC in January 2013 to broaden the focus of surveillance in ventilated patients from pneumonia alone to a larger set of physiologically significant and potentially preventable complications of mechanical ventilation, including pulmonary edema, acute

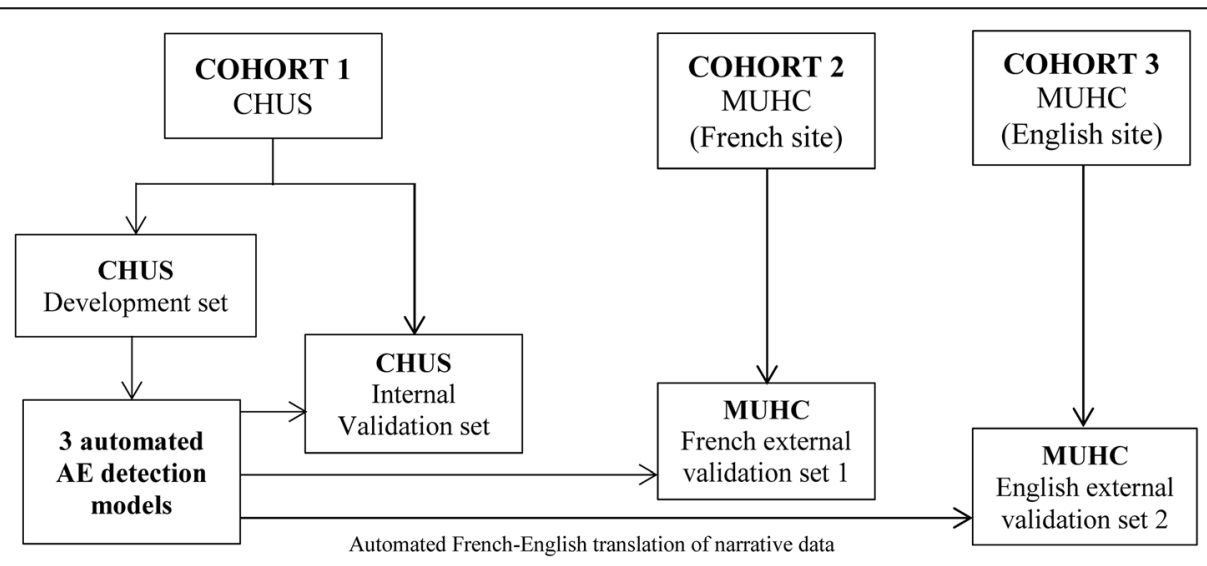

Fig. 1 Project design 

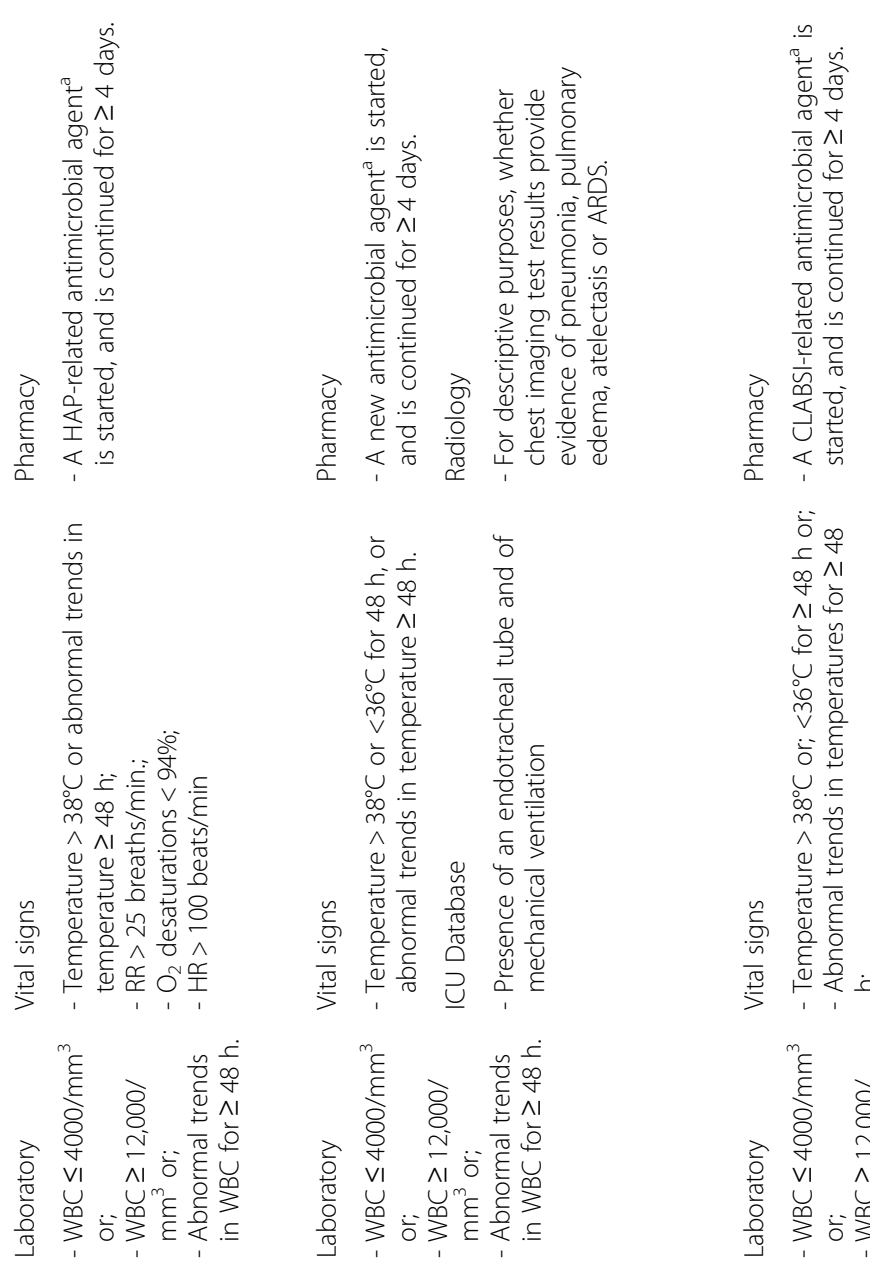

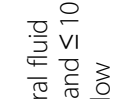
产高

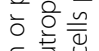

है

흔 눙요

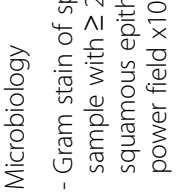
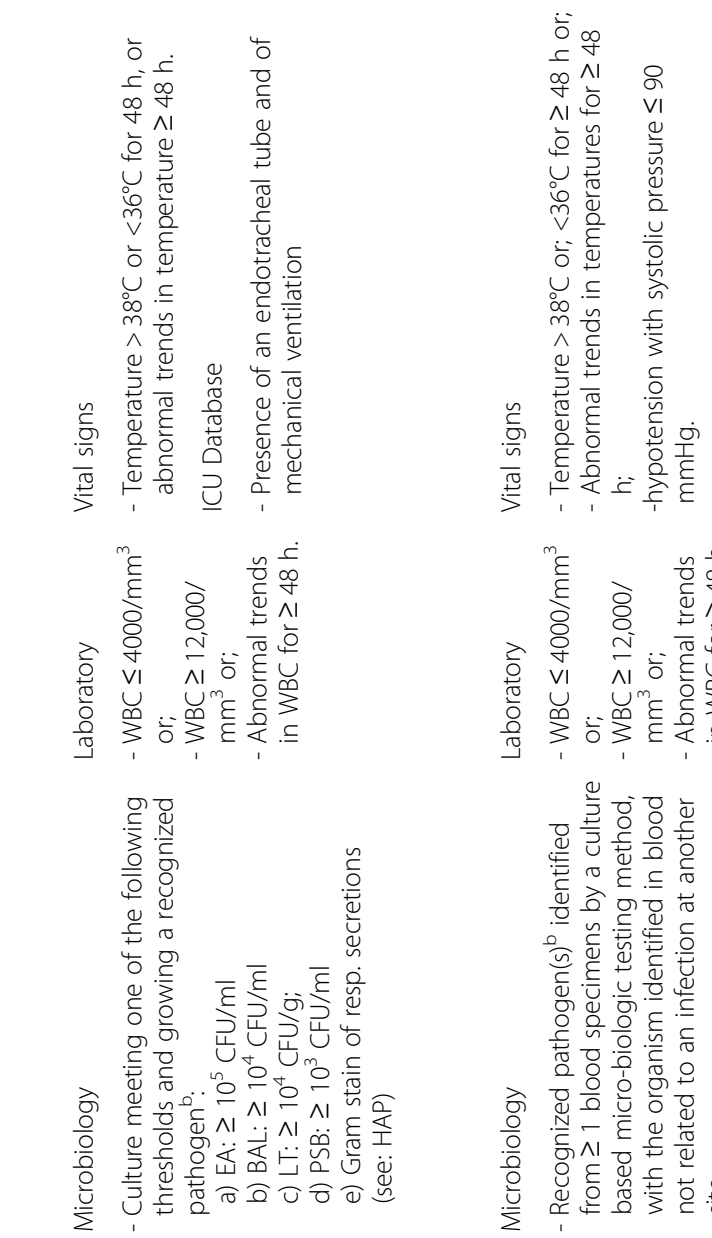

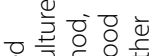

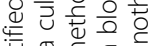

政

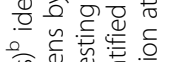

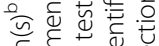

क्ष

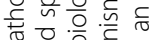

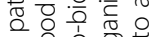

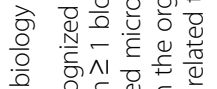

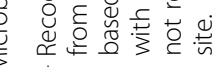


respiratory distress syndrome, and/or atelectasis [28], and, c) central line-associated bloodstream infection (CLABSI) defined as a laboratory-confirmed bloodstream infection occurring in a patient with a central line in place for more than $48 \mathrm{~h}$ on the date that the positive blood culture is identified [23].

These AEs were selected because they are associated with significant morbidity, mortality, and costs [29-31]. Moreover, these indicators have high incidence rates compared to other AEs. HAP accounts for 15\% of all hospital-acquired infections and $25 \%$ of all ICU-acquired infections [30]. HAP is estimated to occur at a rate of 5 to 20 cases per 1000 hospital admissions [30]. VAEs are the most frequent ICU-acquired AEs; occurring in 5.6\% to $10 \%$ of mechanically ventilated patients [31]. Lastly, central lines are the most important cause of bloodstream infections, with CLABSIs occurring in $2 \%$ to $7 \%$ of all catheterizations [32].

\section{Patient demographic and clinical characteristics}

Patient demographic characteristics, comorbidities and severity of illness can influence the likelihood of $\mathrm{AE}$ occurrence, the accuracy of $\mathrm{AE}$ detection models and the generalizability of these models across institutions [11]. Patient age and sex will be extracted from the discharge abstract database. Comorbidities will be measured using the Charlson Comorbidity Index, a weighted index of 17 comorbidities [33]. Comorbidities will be measured at the time of hospital admission using discharge diagnostic codes from prior hospitalizations since 2008 (i.e., the earliest date for which complete data is available at the study sites). Severity of illness in medical and surgical patients will be measured within $24 \mathrm{~h}$ of hospital admission using the Laboratory-based Acute Physiology Score (LAPS); a scoring system that integrates information from 14 laboratory tests into a single continuous variable [34]. Severity of illness in ICU patients will be measured using the Acute Physiology and Chronic Health Evaluation (APACHE) III Score; a scoring system that integrates 12 physiologic measurements [35]. APACHE III scores are systematically measured at the CHUS and the MUHC within 24 h of ICU admission and stored in the ICU database.

\section{Reference standard development and validation}

Charts will be reviewed by trained medical chart reviewers (MCRs) who will perform chart review using standardized surveillance definitions (i.e., CDC/NHSN) [21-23]. MCRs will enter patient AE status (i.e., positive or negative) in an electronic abstraction form that was developed during our pilot work [19, 20]. To assess inter-rater reliability, a random $10 \%$ sample of the medical charts will be blindly reviewed by a second MCR, and intraclass correlation coefficients (ICC) will be computed. ICC values above 0.75 will be judged as excellent [36]. To ensure data quality throughout the study, MCRs will undergo periodic quality assurance monitoring [26].

\section{AE detection model development and optimization}

The automated AE detection models will be developed in accordance with published methodological guidelines $[37,38]$, and will mirror CDC/NHSN surveillance definitions (Table 1) [21-23]. Three successive steps will be followed, which build on and expand on our prior research work in the area [26]. In Step 1, receiver operating characteristic (ROC) curves will be used to determine for selected EHR data sources: a) optimal cut points for defining the presence of an $\mathrm{AE}$ (e.g., using various thresholds for defining an elevated white blood cell count, an abnormal ventilator setting or an elevated body temperature), and b) optimal time window for measuring these parameters (e.g., requiring a single day with an elevated white blood cell count versus two or more consecutive days, requiring only one versus two or more consecutive chest $\mathrm{x}$-rays showing evidence of pneumonia) (Table 1) [26]. In addition, ROC area under the curve (AUC), along with its $95 \%$ confidence intervals (CIs), will be used to assess the accuracy of each individual data source. To analyse narrative data, we will build on NLP techniques developed in our prior research work to identify subsets of words, phrases and patterns in clinical narratives that are significantly associated with the occurrence of each $\mathrm{AE}$ of interest $[19,20]$. In Step 2, three separate multivariate logistic regression analyses-one for each $\mathrm{AE}$ of interest-will be conducted to assess the incremental effect in detection accuracy of combining EHR data sources, using the optimal cut points and measurement windows identified in Step 1 [26]. Stepwise and backward procedures will be used to identify data sources that are significantly associated with AE occurrence [37]. AUCs along with their 95\%CIs will be used to assess the incremental effect in detection accuracy associated with the inclusion of a given data source in the regression model. AUCs across models will be compared [38]. Data sources not significantly associated with AE occurrence will be eliminated from the model [26]. In Step 3, the best regression models identified in Step 2 will be used to assess the incremental effect in detection accuracy of including patient demographic characteristics, comorbidities and severity of illness [26]. Specifically, the AUCs of regression models including these characteristics will be compared to those from the best performing models in Step 2 [38]. During each of the aforementioned steps, estimates of sensitivity, specificity, positive predictive value (PPV) and negative predictive value (NPV), along with their 95\% CIs, will be computed [26]. 


\section{AE detection model validation and update}

The best performing models from the development and optimization steps will be applied to a random sample of the remaining $40 \%$ of CHUS patients (cohort 1, internal validation set) and their performance will be assessed using a reference standard assessment of the medical chart. AUCs from the validation set will be compared to those obtained during the development and optimization steps [38]. Estimates of sensitivity, specificity, PPV and NPV, along with their 95\% CIs will be computed for the best performing models.

To assess the extent to which the best performing models developed and validated at the CHUS can be generalized to other acute care hospitals, they will be applied to a cohort of patients admitted to the MUHC French site (cohort 2, French validation set) as well as to a cohort of patients admitted to the MUHC English sites (cohort 3, English validation set) (Fig. 1). Then, a reference standard assessment of the medical charts will be performed at each site for a random sample of AE positive and $\mathrm{AE}$ negative patients. Prior to applying the models to data from the MUHC English site, French words used as predictors of AE occurrence in the CHUS models will be translated into the equivalent English terms using a validated NLP approach [27]. To determine if there are any significant differences in the performance of the AE detection models across sites, the AUCs obtained from cohort 2 and 3 will be compared to those obtained from the best performing models in cohort 1. Lastly, because it is common for the performances of prediction models to degrade when validated in a new patient population, the intercept and the regression coefficients of the CHUS models will be recalibrated (updated), if necessary, on MUHC data [37].

\section{Sample size requirements}

For the development set, assuming an incidence rate of $5.0 \%$ for both HAP and CLABSI [30,32], and of 7\% for VAE [31] a total of 894 AE positive charts (i.e., 298 HAP,
298 VAE and 298 CLABSI) and 5662 AE negative charts is required to generate a $95 \% \mathrm{CI}$ width of 0.10 around a sensitivity estimate of 0.90 [39]. For the validation sets, we will maximize efficiency by using the automated $\mathrm{AE}$ detection models to oversample $\mathrm{AE}$ positive patients in relation to $\mathrm{AE}$ negative ones [40]. Assuming the aforementioned incidence rates, a total of 639 AE positive (i.e., 237 HAP, 165 VAE and 237 CLABASI) and 3099 $\mathrm{AE}$ negative charts is required in each validation set to generate a $95 \% \mathrm{CI}$ width of 0.10 around a sensitivity estimate of 0.90 that is adjusted for the over-sampling of $\mathrm{AE}$ positive patients [40]. To minimize the costs associated with performing chart review, all AE negative patients in the validation sets will be selected so that they are negative for all three AEs according to the AE detection models.

\section{Discussion}

\section{Current study status}

This study was funded by the CIHR in July 2016. We received research ethics approval from the CHUS and the MUHC in August 2016 and are now ready to initiate data extraction at the CHUS. This study will be conducted over 4 years. The details of the study timelines are provided in Fig. 2.

\section{The anticipated contributions}

This study aims to produce more accurate and efficient measures of AEs. These measures could be used to document the incidence rates of AEs, evaluate the effectiveness of interventions targeted at improving patient safety and monitor progresses through time. In addition, because these measures are automatable, they offer the potential to rapidly scan high volumes of EHR data with minimal human input and at relatively low costs, which represent major gains compared to using manual chart review or prevalence surveys. As a result, human resources currently assigned to $\mathrm{AE}$ surveillance in acute care hospitals could be more productively reallocated to

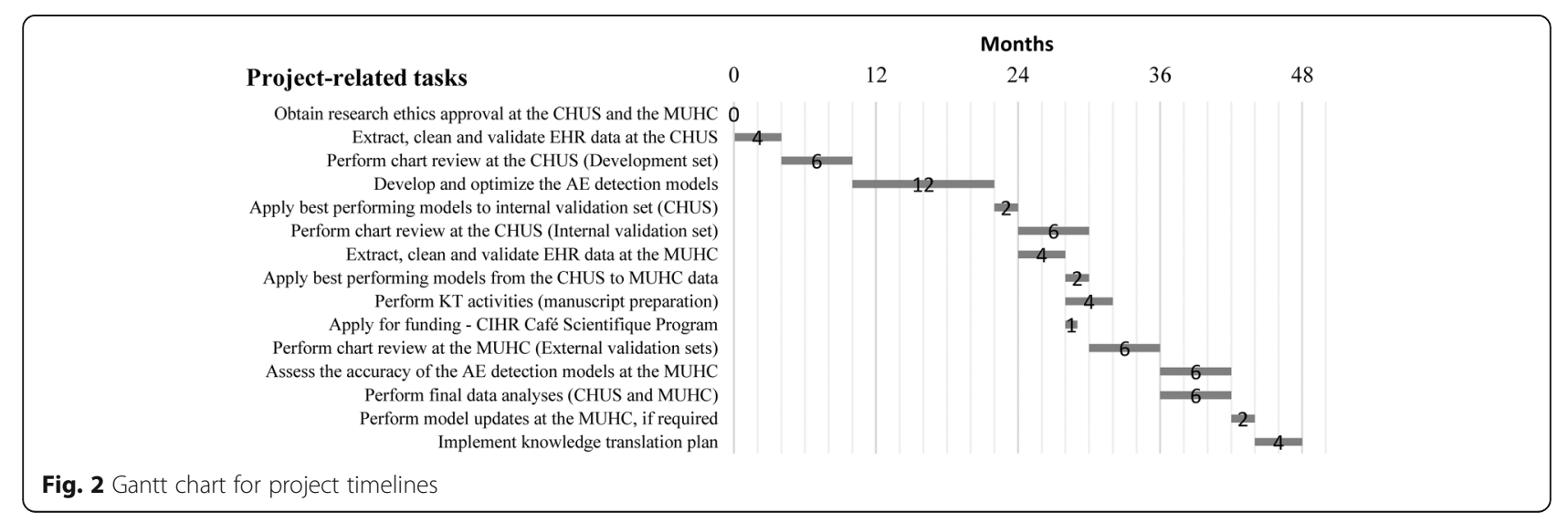


the development and implementation of preventive interventions. Lastly, automation has the potential to standardize AE surveillance; a net gain over manual approaches and a critical requirement to valid interinstitutional comparisons. Such comparisons are needed to define targets for performance improvement, but also to identify and implement best practices from leaders in the field.

\section{Potential challenges and mitigation strategies}

Based on our prior research work at the CHUS and the MUHC, we anticipate three potential challenges. First, EHR data extraction is often delayed by conflicting priorities. To mitigate this challenge, and ensure that the study is conducted within the proposed timelines, we: a) are working on $\mathrm{AE}$ indicators that are highly relevant to the CHUS and the MUHC, b) have invited key decisionmakers from these institutions as co-investigators/collaborators on this study. These decision-makers have authority over the data warehouses at the CHUS and $\mathrm{MUCH}$; the main infrastructure required for supporting the proposed study. They are also important knowledge and technology users; bringing practice-relevant knowledge to the team. Second, while infection preventionists (IPs) routinely monitor HAP, VAE and CLABSI rates, existing data at the study sites is only available for small subsets of selected patients and time periods (as in most other hospitals). Moreover, important variations in the application of surveillance definitions by IPs both within and across hospitals preclude the use of this data as a reference standard [7]. For these reasons, we opted to develop and validate our own reference standard for this study. Last, the performances of prediction models often degrade when they are validated in a new patient population. To guard against this, and maximize the generalizability of the $\mathrm{AE}$ detection models, we have planned for model update techniques in the data analysis steps.

\section{Knowledge translation plan}

To facilitate the dissemination and uptake of the new knowledge that will be generated by this study, our knowledge translation plan will target four groups. First, we have partnered with key decision-makers, clinical leaders and patient safety experts at the CHUS and the MUHC who are engaged as co-investigators/collaborators on the study, have significantly contributed to its development and to the selection of high-priority $\mathrm{AE}$ indicators. Through such engagement, we aim to develop practice-relevant and clinically useful $\mathrm{AE}$ detection models. Moreover, based on the results of our pilot work at these sites $[19,20]$, we are exploring the possibility of integrating the $\mathrm{AE}$ detection models within quality and safety dashboards at the CHUS and the MUHC. These could serve as demonstration projects for other hospitals throughout Canada and abroad. Second, to reach a national audience of potential knowledge users (i.e., patient safety experts, infection control professionals), we will partner with the Communication Services at the University of Sherbrooke to organize and advertise two national webinars (one in French, the other in English) through Mybys web-conferencing technologies (www.mybys.com). These webinars will be tailored to the needs of this audience and will aim to increase awareness about automated AE detection using EHR data, while highlighting key messages and lessons from our research study. Third, we will organize press conferences to inform the population and the media about the findings of this study and the valueadded of EHRs for patient safety. Lastly, we will communicate the findings of this study to academic and research colleagues through conference presentations and submission of manuscripts for publication.

\section{Abbreviations}

AE: Adverse event; APACHE: Acute Physiology and Chronic Health Evaluation: AUC: Area under the curve; CDC/NHSN: Centers for Disease Control and Prevention/National Healthcare Safety Network; CHUS: Centre hospitalier universitaire de Sherbrooke; CIHR: Canadian Institutes of Health Research; CLABSI: Central line-associated bloodstream infection; EHR: Electronic health record; HAP: Hospital-acquired pneumonia; ICC: Intraclass correlation coefficients; ICU: Intensive care unit; IP: Infection preventionist; LAPS: Laboratory-based Acute Physiology Score; MCR: Medical chart reviewers; MUHC: MCGill University Health Centre; NLP: Natural language processing; NPV: Negative predictive value; PPV: Positive predictive value; ROC: Receiver operating characteristic curve; VAE: Ventilator-associated event; VTE: Venous thromboembolism

\section{Acknowledgements}

Not applicable.

\section{Funding}

Funding for this study was provided by the Canadian Institutes of Health Research (CIHR). This funding source was not involved in the design of the study, in the writing of the manuscript, or in the decision to submit the manuscript for publication.

\section{Availability of data and materials}

Data sharing is not applicable to this article as no datasets were generated or analysed in preparation for this manuscript.

\section{Authors' contributions}

All listed authors: 1) have made substantial contributions to conception and design of the proposed study (CR, AB, DB, FD, LV), or acquisition of pilot data $(C R, A B, P L, B P)$, or analysis and interpretation of pilot data that supported the proposed study (CR, BG, AT, FD, SW, TL, DJ, LV, LAA); 2 ) have been involved in drafting the manuscript or revising it critically for important intellectual content (CR, DB, AT, AB, FD, SW, BG, LV, LAA, TL, DJ, BP, PL); 3) have given final approval of the version to be published $(C R, D B, A T, A B, F D$, $S W, B G, L V, L A A, T L, D J, B P, P L)$; and 4) agree to be accountable for all aspects of the work in ensuring that questions related to the accuracy or integrity of any part of the work are appropriately investigated or resolved (CR, DB, AT, AB, FD, SW, BG, LV, LAA, TL, DJ, BP, PL).

\section{Authors' information}

This study brings together an interdisciplinary team of experts who will collaborate in developing the next generation of AE detection systems. Specifically, Dr. Christian Rochefort (RN, PhD) is an Assistant Professor of Nursing at the University of Sherbrooke (UofS) and an Associate Member in the Department of Epidemiology and Biostatistics at McGill. He has expertise in applying NLP techniques to EHR data for detecting AEs. Dr. Benoit Gallix 
$(\mathrm{MD}, \mathrm{PhD})$ is a Professor of Radiology at McGill and the Director of the Diagnostic Radiology Department at the MUHC. He conducts research on the secondary usage of radiology data for quality, safety, and performance improvement. His expertise will be critical to identifying relevant patterns in radiology data during AE detection model development. Dr. David Buckeridge (MD, PhD) is an Associate Professor of Epidemiology and Biostatistics at McGill where he holds a Canada Research Chair in Public Health Informatics. He works on the development and evaluation of surveillance systems that use EHR data. He thus brings highly relevant expertise to the project team. Dr. Shengrui Wang (PhD) is a Professor of Computer Sciences at UofS with expertise in data mining, pattern recognition and machine learning. He has developed ground-breaking techniques for mining high dimensional complex-type data, including advanced statistical models for data clustering and classification. His expertise will be essential to the analysis of narrative data, which is complex and highdimensional. Dr. Andréanne Tanguay (RN, PhD) is an Assistant Professor of Nursing at UofS with extensive expertise in infection control and prevention, which will be critical to adapting the CDC/NHSN definitions for electronic surveillance. Dr. Frederick D'Aragon (MD, PhD) is an Assistant Professor of Medicine at UofS and an intensivist at the CHUS whereas Dr. Dev Jayaraman (MD) is an Associate Professor of Medicine at McGill and an intensivist at the MUHC. Both Dr. D'Aragon and Dr. Jayaraman's expertise will be critical to detecting AEs in ICU patients, especially VAEs. Dr. Alain Biron (RN, PhD) is an Assistant Professor of Nursing at McGill and the Assistant to the Director for Quality, Safety, and Performance at the MUHC where he specializes in quality and performance assessment. Dr. Louis Valiquette (MD, MSc) is a Professor of Microbiology and Infectious Diseases at UofS and the Director of the Department of Microbiology and Infectious Diseases at the CHUS. He has extensive expertise in health informatics, having developed a computerized system for optimizing antimicrobial therapies in hospitalized patients, as well as on nosocomial infections. Mrs. Li-Anne Audet (RN, MSc candidate) is a Master's student specialized in surgical/critical care nursing with research interests in adverse event detection and prevention using information technologies. Her expertise will be essential in interpreting AE data pertaining to surgical and critical care patients. Dr. Todd Lee (MD, MPH) is an Assistant Professor of Medicine at MCGill and an internist at the MUHC where he conducts patient safety research using EHR data. His contribution will be essential to identifying and interpreting AE-related EHR data. Our team also includes decision-makers from the CHUS (Mr. Bruno Petrucci, MBA, Director for Quality and Performance) and the MUHC (Mrs. Patricia Lefebvre, B. Pharm, MSc, Director for Quality and Performance). These decision-makers have respectively authority over the data warehouses at the $\mathrm{CHUS}$ and $\mathrm{MUCH}$; the main infrastructure required for supporting the proposed study. They are also important knowledge and technology users; bringing practice-relevant knowledge to the team.

\section{Competing interests}

The authors declare that they have no competing interests.

\section{Consent for publication}

Not applicable.

\section{Ethics approval and consent to participate}

This study was approved by the Research Ethics Committee at the Centre hospitalier universitaire de Sherbrooke (CHUS) and at the McGill University Health Centre (MUHC). Authorization to access EHR data at the CHUS and MUHC was provided by the Director of professional services (DPS) at these sites in accordance with the provisions of Quebec's Law on Health and Social Services. Given that the proposed study presents minimal risks for the patients, the Research Ethics Committee at the CHUS and MUHC approved a waiver of patient consent.

\section{Author details}

${ }^{1}$ School of Nursing, Faculty of Medicine and Health Sciences, University of Sherbrooke, 3001, 12e Avenue Nord, Sherbrooke, QC J1H 5N4, Canada. ${ }^{2}$ Centre de recherche de l'Hôpital Charles-LeMoyne, University of Sherbrooke-Campus Longueuil, 150 Place Charles-LeMoyne, Longueuil, QC J4K 0A8, Canada. ${ }^{3}$ Department of Epidemiology, Biostatics and Occupational Health, Faculty of Medicine, McGill University, Purvis Hall, 1020 Pine Avenue West, Montreal, QC H3A 1A2, Canada. ${ }^{4}$ Department of Quality, Patient Safety and Performance, McGill University Health Centre, 2155 Guy Street, Montreal,
QC H3H 2R9, Canada. ${ }^{5}$ Ingram School of Nursing, McGill University, Wilson Hall, 3506 University Street, Montreal, QC H3A 2A7, Canada. 'Department of Anesthesiology, Faculty of Medicine and Health Sciences, University of Sherbrooke and Centre hospitalier universitaire de Sherbrooke, 3001, 12e Avenue Nord, Sherbrooke, QC J1H 5N4, Canada. ${ }^{7}$ Faculty of Sciences, Department of Informatics, University of Sherbrooke, 2500 Boulevard de I'Université, Sherbrooke, QC J1K 2R1, Canada. ${ }^{8}$ Department of Diagnostic Radiology, McGill University and McGill University Health Centre, 1650 Cedar Avenue, Montreal, QC H3G 1A4, Canada. 'Department of Microbiology and Infectious Diseases, University of Sherbrooke and Centre hospitalier universitaire de Sherbrooke, 3001, 12e Avenue Nord, Sherbrooke, QC J1H $5 \mathrm{~N} 4$, Canada. ${ }^{10}$ Department of Internal Medicine, McGill University and McGill University Health Centre, 1650 Cedar Avenue, Montreal, QC H3G 1A4, Canada. ${ }^{11}$ Department of Quality, Evaluation, Performance and Ethics, Centre hospitalier universitaire de Sherbrooke, 3001, 12e Avenue Nord, Sherbrooke, QC J1H 5N4, Canada.

Received: 13 September 2016 Accepted: 2 February 2017

Published online: 16 February 2017

\section{References}

1. Kohn LT, Corrigan J, Donaldson MS. To err is human. Building a safer health system. Washington: Institute of Medicine, National Acadey Press; 2000.

2. de Vries EN, Ramrattan MA, Smorenburg SM, et al. The incidence and nature of in-hospital adverse events: a systematic review. Qual Saf Health Care. 2008;17(3):216-23.

3. Zimlichman E, Henderson D, Tamir O, et al. Health care-associated infections: a meta-analysis of costs and financial impact on the US health care system. JAMA Intern Med. 2013;173(22):2039-46.

4. Canadian Patient Safety Institute (CPSI). Patient Safety Forward With Four: The National Integrated Patient Safety Strategy. 2016. http://www. patientsafetyinstitute.ca/en/About/PatientSafetyForwardWith4/Pages/ default.aspx; Accessed 15 Feb 2016.

5. Larizgoitia I, Bouesseau MC, Kelley E. WHO efforts to promote reporting of adverse events and global learning. J Public Health Res. 2013;2(3):e29.

6. van Mourik MS, Troelstra A, van Solinge WW, et al. Automated surveillance for healthcare-associated infections: opportunities for improvement. Clin Infect Dis. 2013;57(1):85-93.

7. Woeltje KF, Lin MY, Klompas M, et al. Data requirements for electronic surveillance of healthcare-associated infections. Infect Control Hosp Epidemiol. 2014;35(9):1083-91.

8. Thonon F, Watson J, Saghatchian M. Benchmarking facilities providing care: an international overview of initiatives. SAGE Open Med. 2015;3: 2050312115601692

9. Talbot TR, Bratzler DW, Carrico RM, et al. Public reporting of health careassociated surveillance data: recommendations from the healthcare infection control practices advisory committee. Ann Intern Med. 2013;159(9):631-5.

10. Halpin H, Shortell SM, Milstein A, et al. Hospital adoption of automated surveillance technology and the implementation of infection prevention and control programs. Am J Infect Control. 2011;39(4):270-6.

11. Govindan M, Van Citters AD, Nelson EC, et al. Automated detection of harm in healthcare with information technology: a systematic review. Qual Saf Health Care. 2010;19(5):e11.

12. Freeman R, Moore LS, Garcia AL, et al. Advances in electronic surveillance for healthcare-associated infections in the 21st Century: a systematic review. J Hosp Infect. 2013;84(2):106-19.

13. Classen DC, Resar R, Griffin F, et al. 'Global trigger tool' shows that adverse events in hospitals may be ten times greater than previously measured. Health Aff (Millwood). 2011;30(4):581-9.

14. Goto M, Ohl ME, Schweizer ML, et al. Accuracy of administrative code data for the surveillance of healthcare-associated infections: a systematic review and meta-analysis. Clin Infect Dis. 2014;58(5):688-96.

15. Groene $\mathrm{O}$, Kristensen $\mathrm{S}$, Arah $\mathrm{OA}$, et al. Feasibility of using administrative data to compare hospital performance in the EU. Int J Qual Health Care. 2014;26 Suppl 1:108-15.

16. Hammond W, Jaffe C, Cimino J, Huff S. Standards in biomedical informatics. In: Shortliffe E, Cimino J, editors. Biomedical informatics: Computer applications in health care and biomedicine. 4th ed. New York: Springer; 2014. p. 211-54.

17. Ohno-Machado L. Realizing the full potential of electronic health records: the role of natural language processing. J Am Med Inform Assoc. 2011;18(5):539. 
18. de Bruin JS, Seeling W, Schuh C. Data use and effectiveness in electronic surveillance of healthcare associated infections in the 21st century: a systematic review. J Am Med Inform Assoc. 2014;21(5):942-51.

19. Rochefort $C M$, Verma AD, Equale T, et al. A novel method of adverse event detection can accurately identify venous thromboembolisms (VTEs) from narrative electronic health record data. J Am Med Inform Assoc. 2015;22(1):155-65.

20. Tian Z, Sun S, Eguale T, et al. Automated Extraction of VTE Events From Narrative Radiology Reports in Electronic Health Records: A Validation Study. Med Care. 2015 Apr 28.

21. Centers for Disease Control and Prevention. Pneumonia (Ventilatorassociated [VAP] and non-ventilator-associated pneumonia [PNEU] events. 2015. http://www.cdc.gov/nhsn/PDFs/pscManual/6pscVAPcurrent.pdf; Accessed 16 Jan 2016.

22. Centers for Disease Control and Prevention. Ventilator-associated events (VAE). 2015. http://www.cdc.gov/nhsn/PDFs/pscManual/10-VAE_FINAL.pdf; Accessed 8 Jan 2016.

23. Centers for Disease Control and Prevention. Bloodstream Infection Event (Central Line-Associated Bloodstream Infection and Non-central lineassociated Bloodstream Infection). 2015. http://www.cdc.gov/nhsn/PDFs/ pscManual/4PSC_CLABScurrent.pdf; Accessed 8 Feb 2016.

24. Le Centre hospitalier de I'Université de Sherbrooke (CHUS). Le Centre hospitalier de I'Université de Sherbrooke (CHUS). 2015. http://www.chus.qc. ca/le-chus/centre-hospitalier-universitaire/; Accessed 8 Feb 2016.

25. McGill University Health Centre (MUHC). MUHC at a glance. 2014. http:// muhc.ca/homepage/page/muhc-glance; Accessed 19 Nov 2014.

26. Rochefort CM, Buckeridge DL, Forster AJ. Accuracy of using automated methods for detecting adverse events from electronic health record data: a research protocol. Implement Sci. 2015;10:5.

27. Lei S, Mihalcea R, Tian M. Cross Language Text Classification by Model Translation and Semi-Supervised Learning. Massachusetts: MIT; 2010. p. 1057-67.

28. Magill SS, Klompas M, Balk R, et al. Developing a new, national approach to surveillance for ventilator-associated events*. Crit Care Med. 2013:41(11): 2467-75.

29. Stevens V, Geiger K, Concannon C, et al. Inpatient costs, mortality and 30day re-admission in patients with central-line-associated bloodstream infections. Clin Microbiol Infect. 2014:20(5):0318-24.

30. Barbier F, Andremont A, Wolff M, et al. Hospital-acquired pneumonia and ventilator-associated pneumonia: recent advances in epidemiology and management. Curr Opin Pulm Med. 2013;19(3):216-28.

31. Klompas M, Kleinman K, Murphy MV. Descriptive epidemiology and attributable morbidity of ventilator-associated events. Infect Control Hosp Epidemiol. 2014;35(5):502-10.

32. Fontela PS, Platt RW, Rocher I, et al. Epidemiology of central line-associated bloodstream infections in Quebec intensive care units: a 6-year review. Am J Infect Control. 2012:40(3):221-6.

33. Sundararajan V, Henderson T, Perry C, et al. New ICD-10 version of the Charlson comorbidity index predicted in-hospital mortality. J Clin Epidemiol. 2004;57(12):1288-94

34. Escobar GJ, Greene JD, Scheirer P, et al. Risk-adjusting hospital inpatient mortality using automated inpatient, outpatient, and laboratory databases. Med Care. 2008;46(3):232-9.

35. Knaus WA, Wagner DP, Draper EA, et al. The APACHE III prognostic system. Risk prediction of hospital mortality for critically ill hospitalized adults. Chest. 1991;100(6):1619-36.

36. Chapman WW, Dowling JN, Wagner MM. Generating a reliable reference standard set for syndromic case classification. J Am Med Inform Assoc. 2005; 12(6):618-29.

37. Collins GS, Reitsma JB, Altman DG, et al. Transparent Reporting of a multivariable prediction model for Individual Prognosis or Diagnosis (TRIPOD): the TRIPOD statement. Ann Intern Med. 2015;162(1):55-63.

38. Pepe M. The statistical evaluation of medical test classification and prediction. New York: Oxford Press; 2004.

39. Flahault A, Cadilhac M, Thomas G. Sample size calculation should be performed for design accuracy in diagnostic test studies. J Clin Epidemiol. 2005;58(8):859-62.

40. Irwig L, Glasziou PP, Berry G, et al. Efficient study designs to assess the accuracy of screening tests. Am J Epidemiol. 1994;140(8):759-69.

\section{Submit your next manuscript to BioMed Central and we will help you at every step:}

- We accept pre-submission inquiries

- Our selector tool helps you to find the most relevant journal

- We provide round the clock customer support

- Convenient online submission

- Thorough peer review

- Inclusion in PubMed and all major indexing services

- Maximum visibility for your research

Submit your manuscript at www.biomedcentral.com/submit 